\title{
Nonconventional Machining and Materials Processing: Proposed Design for Abrasive Jet Machining (AJM)
}

\author{
Laura Schaefer \\ Department of Mechanical Engineering, Rice University, Main St., Houston, TX, USA \\ laurascha_rice@hotmail.com
}

\begin{abstract}
Article Info
Journal of Machine and Computing (http://anapub.co.ke/journals/jmc/jmc.html)

Doi : https://doi.org/10.53759/7669/jmc202101001

Received 10 October 2020; Revised form 20 November 2020; Accepted 25 December 2020.

Available online 05 January 2021.

(C)2021 The Authors. Published by AnaPub Publications.

This is an open access article under the CC BY-NC-ND license. (http://creativecommons.org/licenses/by-nc-nd/4.0/)

Abstract - High degree Abrasive Jet (AJ) is applied in Abrasive Jet Machining (AJM) as a process that is based of Machine-driven Energy (MDE). The abrasives are applied in the removal of resources from work surfaces based on impact erosion. AJ is generated through the acceleration of very fine abrasive particles in considerably gas that has been pressurizes i.e. carrier gas. In this case, jets are utilized to transform this pressurized energy into kinetic energy, which is also directs jets to the work surfaces at an impingement angle. During the impact, rigid abrasive particles are used to slowly remove resources through erosion and with the assistance of brittle fracture. This paper evaluates and recommends a design for AJM. The various components have also been chosen after the relevant design assessment procedures have been done. The AJM model has been design with reference to the present components.
\end{abstract}

Keywords - Abrasive Jet (AJ), Abrasive Jet Machining (AJM), Machine-driven Energy (MDE), Material Removal Rate (MRR).

\section{INTRODUCTION}

Unconventional processes of manufacturing represent the processes that are used in the removal of excess resources [1]. These processes involve chemical, electrical, thermal and mechanical energy or a combination of these energies. This paper will focus on Abrasive Jet Machining (AJM). AJM provides a process of removing resources of high degree abrasive resources with the assistance of gas from jets. The elimination of resources is initiated through the process of erosion. AJM is majorly utilized in the cutting of shapes in brittle and hard resources e.g. ceramic and gas where Machine-driven Energy (MDE) is used to automate machine with three-axe travel. The various AJM components include dust filters, stress regulators, dehumidifier, vibrator, vertical motion module i.e. z-motion, horizontal motion modules i.e. $\mathrm{x}, \mathrm{y}$ board.

The process of AJM is different from the conventional blasting of sand whereby abrasive is considerably fine and actions of cutting and procedural constraints are critically controlled. The process is utilized for cleansing and deburring processes. AJM is characteristically free from vibration and chatter issues. The act of cutting is finer due to the objective of the carrier gas in cooling. AJM is different from the traditional sand blasting methodology by the attainable dimension of precision and accuracy [2]. AJM makes use of different abrasives incorporate sodium bicarbonate, glass beads, silicon carbide, and alumina while sand blasting majorly makes use of silica sand. Even though main aims of the two processes are the same, the constraints of cutting can be guided in AJM and therefore it can provide the best form of precision and accuracy.

In the process of AJM, it is viewed that rigid abrasive particles are used in the elimination action whereas the carrier gas helps in blowing the eroded resources from the machining region. These abrasives have to incorporate basic features for effective elimination of resources and getting the required cut quality. Among the desired features effective hardness, presence of sharpened ends, irregular shapes and effective flow features are fundamental.

Various abrasives with grit sizes can be used with reference to the workpiece resources and the processes it is projected to do. Alumina of the mean size $10 \mu \mathrm{m}$ to $50 \mu \mathrm{m}$ is typically utilized for drilling and grooving processes mostly when work resources are rigid. Nonetheless, for each rigid work material, Silicon Carbide is used since it is more rigid compared to alumina. For coating and polishing removal objectives, crashed glass and glass heads are conventionally utilized [3]. 
Abrasive sizes affect the cut quality, which incorporates the Material Removal Rate (MRR). Large grit sizes tend to generate large cavities and therefore MRR enhances with surface polish. Contrary to that, finer abrasives minimize MRR hut enhance the surface accuracy and quality [4]. Major objective of the carrier gas in Abrasive Jet (AJ) is to accelerate the finer particles through the transfer of momentum. Compressors are utilized to elevate carrier gas stress i.e. more than 20 bar; abrasive grit are mixed within it in mixing chambers (according to the mixing ratio). Jets are utilized to change pressurized MDE i.e. in the form of high speed AJ. Carrier gas compression alongside with jets diameter determines the last jet speed and therefore the performance of machining.

Among different gases, air is typically utilized in AJM as it present in abundance pure nitrogen and carbon dioxide are utilized to harness the best performance for a certain purpose. Nonetheless, clean oxygen is not utilized as it can promptly oxidize the working surface. Before compressions, carrier gases are dehumidified effectively as the presence of steams can block pipelines. Whenever gases are compressed to high stress, steam may cool and tiny water particles can form large globule after agglomerating with AJ. The carrier gas is made free from dust before being compressed to high stress.

The main function of jets in AJM is to change MDE to pressurized abrasive-gas mixture based on kinetic energy as high speed AJ. Jets also direct high speed AJ to the work surfaces from particular distances, i.e. Standoff Distance (SD), and at certain pre-defined angle i.e. impingement angle [5]. The inner diameter of the jets is considered the paramount parameter since it determines the final speed and cross-sectional areas of the jet for a particular gas stress. When the rate of flow and the compressors of stress are constant, the speed of AJ will be inversely proportional to the jet area.

The choice of the appropriate jet is another factor to consider from an economic perspective. Typically, sapphire jets and tungsten carbide are utilized in mechanical firms. Tungsten carbide is more affordable but has less life i.e. limited to 1-2 days. On the other hand, sapphire jets has more life i.e. limited to 6-8 days but expensive. Frequent transformations of jets are linked to the idle time during the process of machining. There are various factors, which can affect the performance of AJM. The essential AJM constraints incorporates work resources' stress concentration and mechanical features; jets' interior diameter and profile; AJ's stand-off distance, impingement angle, striking speed and mixing ratio; carrier gas temperature, stress, flow rate, composition and nature; and abrasive particles flow rate, strength, material, size and shape.

The performance of AJM is typically assessed based on the analysis of three output responses: roughness of the surface and accuracy of machined characteristics; MRR, and rate of jets wear. As detailed earlier, flow rate, material, strength, size and shape of abrasive can affect the performance of machines. The irregularly shaped AJ with sharp ends produce higher MRR than the round grits. The grits with a smaller size provide high finished surfaces but minimize MRR and therefore productivity diminishes. Large grits can be problematic during the process of flowing and mixing through pipelines. Nonetheless, size variation in the complete volume has to be minimal otherwise the assessments and estimations will not be accurate. Abrasive resources have different rigidity. The more rigid an abrasive is based on the rigidity of work surface, the larger the volume of MRR. It is fundamentally that the relative rigidity between workpiece and abrasives determine machines productivity and capacity.

The introductory section has introduced the subject of AJM and explained the various constraints and components of the AJM process. The later sections of this research explain the design for AJM. Thus, the remaining sections of this paper are organized as follows: Section II discusses the background analysis of the proposed model. Section III evaluates the relevant literature about AJM. Section IV presents a critical analysis of the proposed design. Section V presents the final design while Section VI concludes the paper and presents future paths on AJM and its automations.

\section{BACKGROUND ANALYSIS}

\section{A. Rigidity and Stress of AJM}

The aspect of rigidity bases on the arched and bowl-shaped geometrical illustrations of depths that are below $29 \mathrm{~mm}$. This also incorporates the production of thinner walls and slots with depths more than $429 \mathrm{~mm}$ and thickness less than 3 $\mathrm{mm}$. The intricate profile, which denotes the radii succession, is based on the variation of the grinded wheel. This shows the ability of AJM to turn and achieve the objectives of the design in engineering. The machines procedure generates no heat energy and the therefore transitions in strength and microstructure of surfaces are unlikely. Air is a coolant and therefore AJM has higher potential as damage-free micro-machines approach.

The fracture rigidity and toughness of the targeted particles are major constraints influence the MRR in AJ. Apart from that, their effect of machines varies fundamentally based on the applicable abrasives. For some time now, AJ has attained enhanced deburring effectiveness in engineering applications. The effects of AJ constraints has not been identified clearly. Deburring in this case is of significant over the physical methodology of deburring because it can produce the end radii in an automated manner. It develops the quality of the deburring components.

The process of burr elimination and convex end production is considered varying as an element of the constraints jet heights and the impingement angles based SOD. The influence of constraints; abrasive size, mixing ratio, and jets stress are 
less significant. SOD was considered a factor that is most influential on the radii size produced at the ends. The end radii produced was considered to be limited to burr root depth. Abrasive jet finish linked with grinding provides the precision finishing procedure known as the integration manufacturing initiatives whereby slurry of liquid solvent and abrasives are incorporated to the grinding region between the work surface and grinding wheel under no radii feeding condition. The abrasive material is powered by the rotating grinding liquid and hydrodynamic stress. Wheels based on enhanced slurry speed between the work surfaces and the grinding wheel to attain micro-removal finishing.

Abrasive water jet machine is becoming globally used in mechanical engineering. Machines provide a significant advantage in the engineering of complex geometrical parts in wide-range resources. The capacity to machine hard resources considering the developments in both software and hardware utilized in water jet machines has influenced technologies to spread widely and used in the engineering sector. Novel advancements in high-stress pumps assure more hydraulic energy at its cut heads hence advancing the performance of the cut of these machines.

Assessments of technical and economic researchers argue that the technological advancements apply high-energy machines and smart software controls to enhance the general performance of abrasive water jet machines processes. As a result, this widens the scope of the potential application of the promising and innovative technology. The surface quality generated during the machines process of aluminum has been evaluated over the past few years. The form of abrasive utilized was garnet of mesh (sized 80). The variables of cuts were standoff jets distances from the work surfaces, jet stress and work feed rates.

The assessment method of the surface generated were cut width, tapers of the cutting slots and rigidity of the work surfaces. It was noted that to minimize the cut width, jets have to be incorporated close to the work surface. The enhancement of stress in jets leads to the widened cut slot at the top and bottom of jet's work surfaces.

Apart from that, the depth of the cut at the bottom part i.e. the exit was viewed to be larger than the standoff depth at the top and approximately $2 \mathrm{~mm}$ based on the work feeds' rate of approximately $14 \mathrm{~mm}$ per min. It was therefore noted that the cutting taper gradually reduces the enhancement of the standoff depths that are closer to 0 and the distances of about $5 \mathrm{~mm}$ (ie at stresses of $14 \mathrm{~mm}$ per min and $29 \mathrm{ksi}$ of the work feeds' rate). The feeds' rate needs maintenance within $39 \mathrm{~mm}$ per min at distances of $4 \mathrm{~mm}$ and stresses of $29 \mathrm{ksi}$. This is because of the feeds' rate that surpasses $39 \mathrm{~mm}$ per min hence amounting to a sharper development in the taper angles.

The stress of the jet does not indicate fundamental impact on the taper's angle in the dimension of the work feed's rate show abrasive impact on the roughness of the machine surface. As such, standoff depths need maintenance within 2 mm (based on the stress released on jets of $29 \mathrm{ksi}$ and the work feeds' rate of $14 \mathrm{~mm}$ per min). The work feed's rate should be referenced to $29 \mathrm{~mm}$ per min, which projects the stress at the jet of $29 \mathrm{ksi}$ and standoff depth of $2 \mathrm{~mm}$. This will therefore facilitate significant finishing since the values of parameters are the best, and there is rigidity of the machined surfaces rising significantly.

The development in jet stress indicates positive impact based on the smoothness of the machined surfaces. With the advancement in the stress from the jets, the surface roughness diminishes i.e. at the work feeds' of $14 \mathrm{~mm}$ per min and the standoff depth of $2 \mathrm{~mm}$. This is because to the fragmentation of the abrasive resources into minor sizes at high stress and because of minor particles producing smooth surfaces. In that case, within the stress of jets considered, the work surfaces are smooth next to the upper surfaces and gradually it becomes rigid at the upper depth.

\section{B. Equipment Involved}

Fig 1 below shows a schematic presentation of AJM. The filtered gas, which is generated under stress to the mixing segments including the abrasive energy and vibrates at $50 \mathrm{c} / \mathrm{s}$, includes abrasive particles and it incorporated into the connecting hose. The gas and abrasive mixture emerges from the minor jets at high speed. Abrasive powder feeding rate is governed by the vibration amplitude of the mixing segment. Jets are mounted on fixtures.

Cams pantograph or effective mechanisms are used to govern the shape and size of the cuts shift either the jets or workpiece. The hand processes are enough to eliminate surface cutting or contamination where accuracy is not fundamental. The equipment for removing dusts is essential to safeguard the ecosystem. Commercialized bench-mounting units incorporating the dust controls, motion generating devices and control devices are present.

\section{Components}

The components include workpiece, vertical \& horizontal motion modules i.e. for xyz motions, mixer \& vibrator, stress regulators, stress gauge, dehumidifier, air filters and air compressor.

\section{AJM Variables}

The variables, which affect MRR and machines accuracy are: cut shape \& operation form; jets working distance (sand distance); geometry material and jets composition; work resources; rate of abrasives; AJM speed; sizes of abrasive grain; forms of abrasive; and carrier gas. 


\section{E. Advantages \& Disadvantages}

The advantages includes being the capacity to cut intricate shapes in holes available in resources based on brittleness and rigidity; capacity to cut soft resources and those sensitive to heat without damages; no transformation in microstructure as no heat energy is produced in AJ; and affordability.

Disadvantages include MRR being minimal and its applications limited. Secondly, stray string can happen and thus its application is considered limited; embedding of abrasives in the surfaces of workpiece may happen when machines soft resources; abrasive resources may accumulate at jets and fail the procedure in case water is not available in the atmosphere; and it cannot be utilized in the drilling of blind holes.

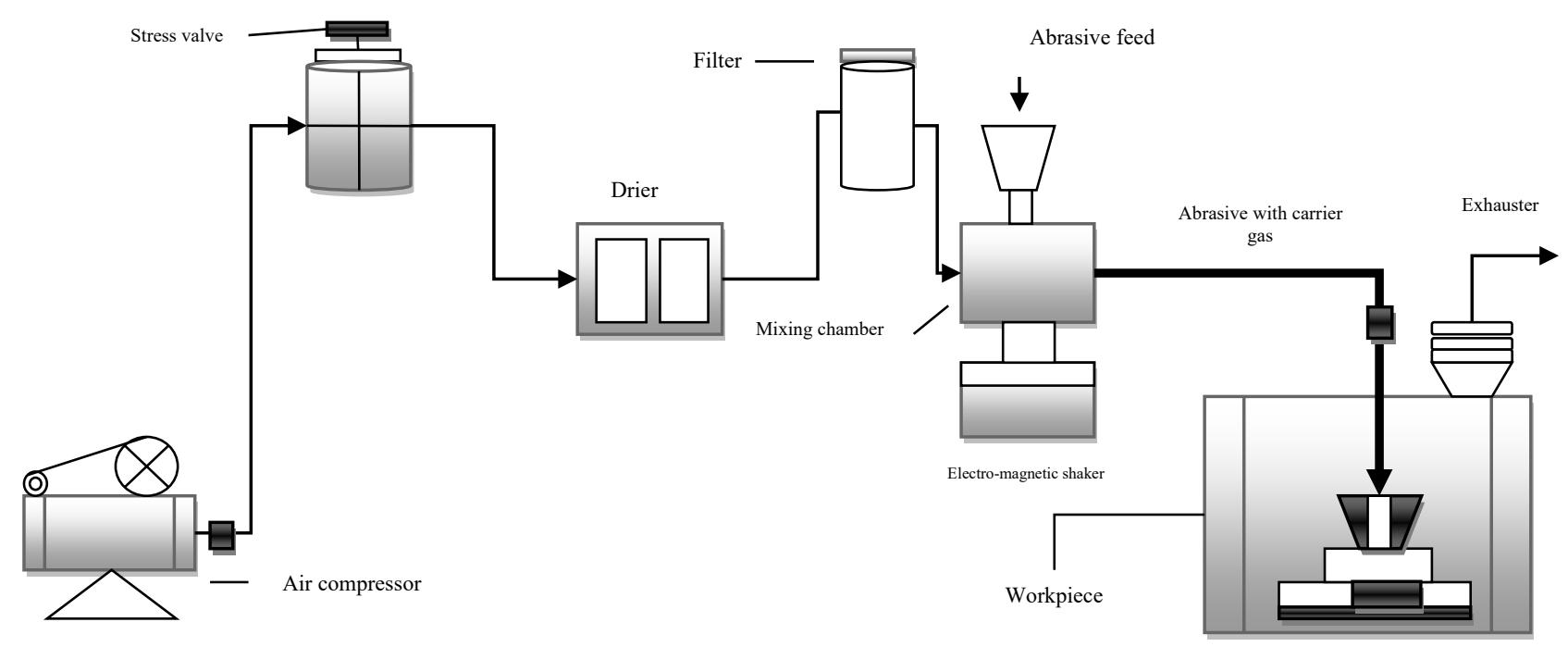

Fig 1: Schematic presentation of AJM

\section{F. Application}

The main fields of AJM application is in the engineering of critical brittle materials, which are heat sensitive e.g. ceramics, mica, semi-conductor resources, sapphire, quartz and gas. It is utilized in the cut slot, deburring, drilling, countering, and thin sections for generating incorporated shapes in brittle and rigid resources. It is typically utilized for polishing and cleaning of plastics Teflon and nylon components. Fragile cleansing such as the elimination of smudges from the antique texts is possible with the process.

\section{LITERATURE REVIEW}

Y. Ali, P. Mathew and J. Wang in [6] argue that AJM presents the machines processes that began a few years ago. Until now, there has been a thorough theoretical and practical analysis on AJM. Numerous research assumption argue about hydrodynamic features of AJ, which ascertains the influences of operational variables on AJM's effectiveness incorporating abrasive concentration, size and type, influence speed and impingement angle. Other researchers identified novel issues about carrier gas typologies, jets size and shape, AJ speed and SD.

M. Kaladhar in [7] evaluated the general performance for the process based on MRR, surface finishing and geometrical tolerance of the work piece, including the ware rate of jets. Lastly, various important literature texts concentrate on the leading aspects of mechanisms in machines of the brittle and ductile resources or concerning the enhancement of systematic statistical-experimental techniques and Artificial Neural Networks (ANN), which facilitates the linking between the procedural setting of variables and the accuracy of machines based on the surface finish rate.

J. Delgadillo and R. Rajamani in [8] assessed the computational fluids dynamics of procedural formation and discharges of airflow and liquid in abrasive water jet. Vibrant features of the flow in abrasive water jet from the jets has been simulated based on turbulent, steady states and phased flow condition. The ultimate objective is to achieve critical assumptions regarding the ultrahigh speed and flow of vibrant features, which might influence the jet quality i.e. stress and speed distribution in various forms of abrasive water jet and the various outlets.

$\mathrm{R}$. Li et al. in [9] underpinned experiments have been done on the influence of jet stress, rate of abrasive flow and work feed rates regarding the rigidity of the surface by abrasive water jet machines i.e. grade p25 carbide. Grade p25 carbide is rigid and is not machined using conventional methods. Abrasives utilized in analysis were mesh garnets of size 79. Carbide was cut with lower and medium levels of abrasive flow rate. Nonetheless, the cutting of carbide in jets was unsuccessful due to the rigid and high degree energy. Minimal abrasive flow rate that was permitted to enhance the cutting process of carbide was 134 gram per min. 
J. Fair in [10] argued that with the development in jet stress, surfaces are smooth because of higher kinetic power of abrasives. Nonetheless, the surface next to the entrance of jets is smoother and the surface is slowly becoming rougher and surfaces slowly becoming rough downwards and roughest close to the jet exit. The increment in the flow rate of abrasives makes the surfaces smooth since this because to the available highest number of cut end per unit segment in a given time. The rate of feed does not indicate the fundamental influence on the machine surfaces; however, it was not noted that the surface rigidity enhances drastically close to the entrance of jets.

P. Jeyapandiarajan and X. Anthony in [11] presented an analysis of results of machines based on different conditions considers that commercial AJM machines was utilized based on the diameter of jets that range from $0.5 \mathrm{~mm}$ to $0.7 \mathrm{~mm}$, the resource of jets is tungsten sapphire and carbide with longer system life. Silicon carbide and aluminum oxide are the dual abrasive utilized. Additional constraints evaluated include jets tip distance ( $5 \mathrm{~mm}$ to $10 \mathrm{~mm}$ ), stress ( 5 bars and 7 bars) and spray angles $\left(60^{\circ}\right.$ and $\left.90^{\circ}\right)$ for resources such as Electronic Discharge Machine (EDM), ceramics and glass steel. The drilling of holes is done based on the application of AJ and might be circular or cylindrical; however, it is entirely elliptical and mounted on a bell. Higher MRR settings do not necessitate the production of narrow and minor clean cut machine parts.

H. Nouraei, A. Wodoslawsky, M. Papini and J. Spelt in [12] show that AJ is relatively an attractive micro machining method for ceramic materials. Machining during the produce of machining AJ can be related to well-defined models of solid material erosion, whereas resource elimination is viewed to come from the crack formation framework. Apart from that, it is viewed that erosion system are never unavoidably applied to machining of AJ tests and findings. This is due to relative rigidity of abrasive over the targeted material that is not considered in the model. This is fundamental in the micromachines procedure.

N. Yuvaraj and M. Pradeep Kumar in [13] argue that there is minimal rigid degradation occurring for machining of AJ ceramic surface. This is relative to the radial crack, which is never propagated through the impact of particles during the process of machines. Abrasive water jet turning is a technological that focusses on finding niche fields of applications whereby it is economically viable. However, a certain application of abrasive water jet turning has demonstrated its economical and technology capacity, i.e. Profiling and covering of the grind wheel. Starting from theoretical assumptions, the main operational parameters of abrasive water jet turning are incorporated in an approach to produce different profiles of the grinding wheel through tangential movement of jet plume.

\section{CRITICAL ANALYSIS OF THE DESIGN}

This section presents the design of AJM as displayed in Fig 2, Fig 3, Fig 4 and Fig 13. The subsections discuss the design components, LMguidepath, and the jets for the AJM.

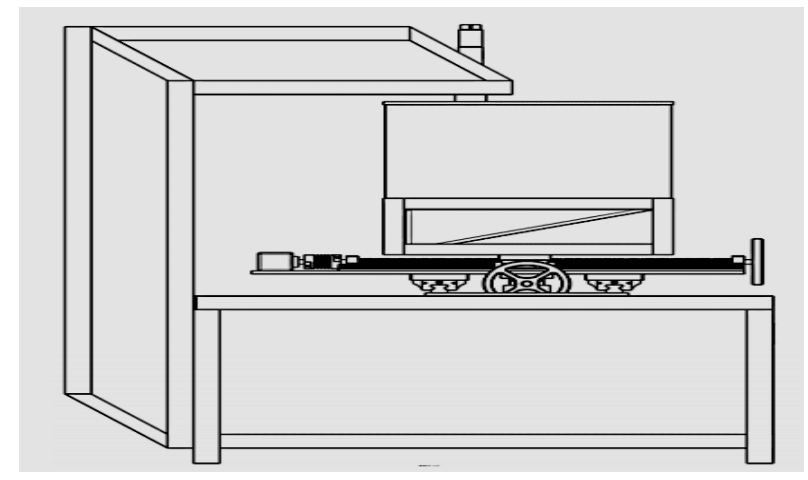

Fig 2: Design assembly (side-view)

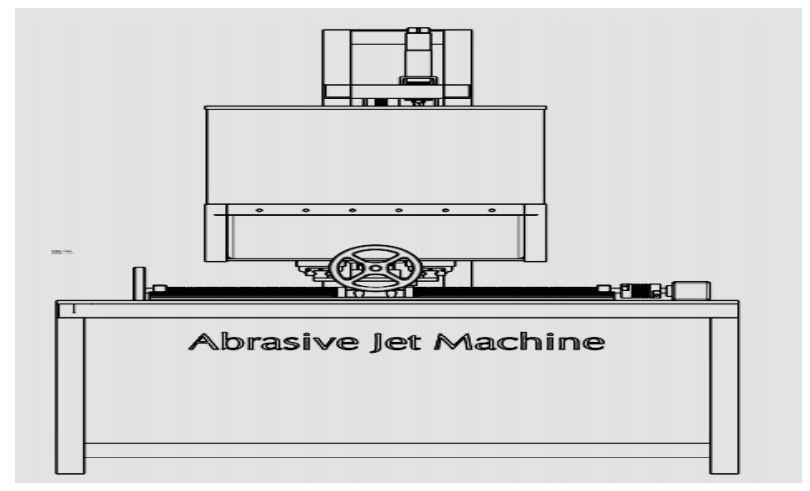

Fig 3: AJM design assembly (front-view) 


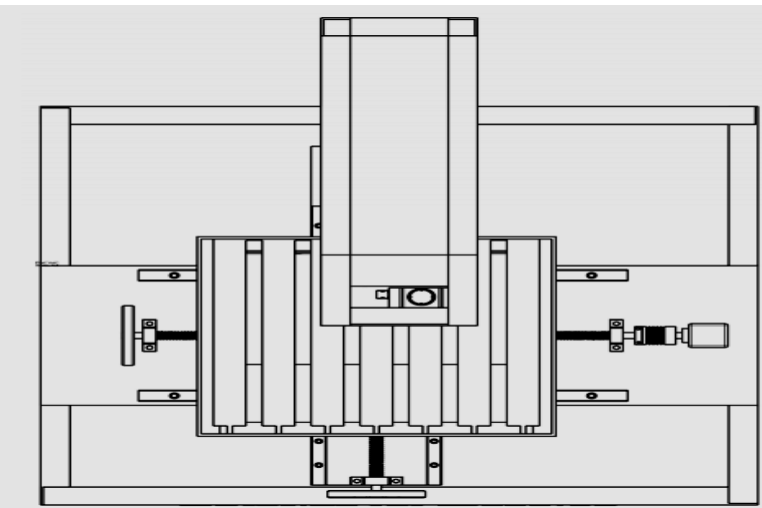

Fig 4: Final AJM design assembly (top-view)

\section{A. Design of Components}

\section{1) The xy Board}

The xy board is the most fundamental segment of AJM whereby the workpice is machined and kept. The xy table and travel should be determined as 400 by $350 \mathrm{~mm}$. The various design component of xy board are:

- $\quad$ LM guidepath i.e. 4 nos and 2 pairs;

- $\quad$ Ball screws i.e. 2 nos;

- $\quad$ Supportive units i.e. 2 supports and 2 fixes;

- $\quad$ Nut brackets i.e. 2 nos;

- $\quad$ Couples i.e. 2 nos

- Standardized bolts and nuts

- Other design components i.e. those to manufactured in the laboratory.

The xy board incorporates two segments: The top table and the bottom table. The top table is obliged to initiate the xmovemnent and travels in $400 \mathrm{~mm}$. The bottom table travels within $350 \mathrm{~mm}$ and it obliged to initaite the y motion of workpieces. The various diagrams of the assembeled views alongside the dimensions have been indicated below (Figs 5, 6 and 7).

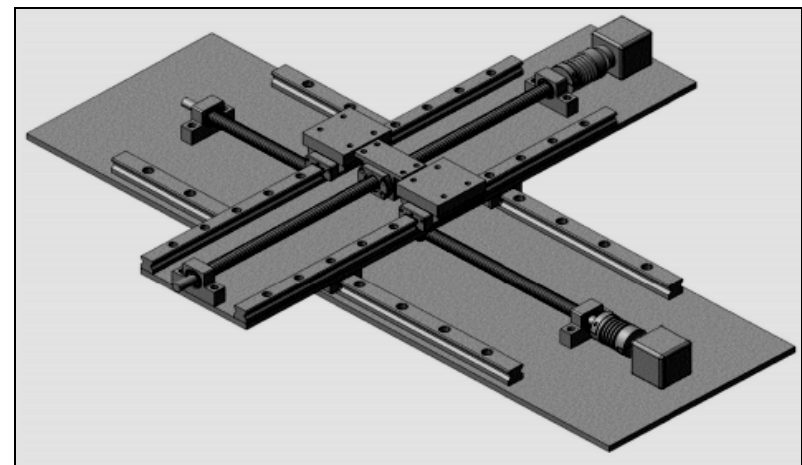

Fig 5: The isometric view of xy board assembly

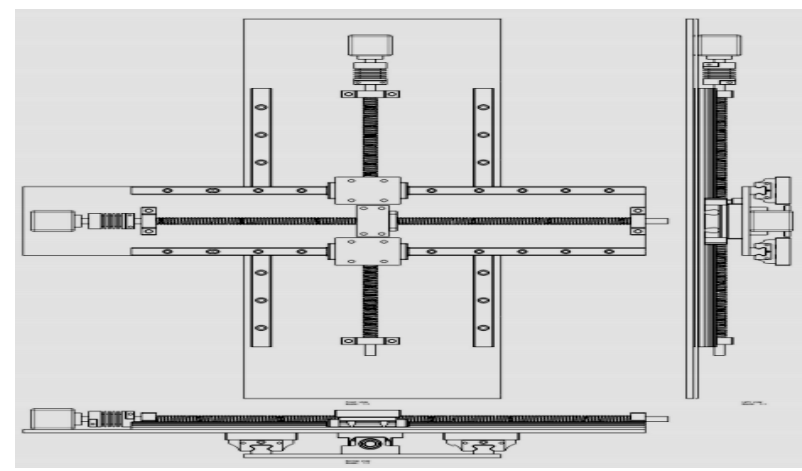

Fig 6: 2D views of the xy board assembly 


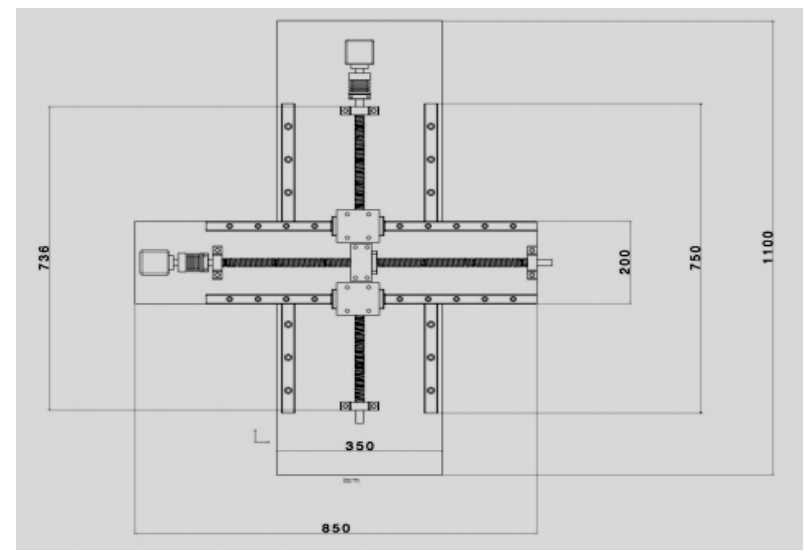

Fig 7: The top view of the of the xy board assembly

2) Ball Screws

The ball screws as shown in Fig 8 used are the same as the lead screws based on the dimensions given in Fig 9. However, the friction in them is significantly minimized for the ball screw by including the circulating ball in the thread and screw nut. The threading profile is viewed as circular and nut thread is considered to be reverse in shape and forms spiral holes whereby the balls move when the nut are rotated. When the ball reaches the end of nuts, they are recirculated using the return pipe. The ball screw can non-preload or preload. The accuracy aspect advances when the preloaded form of the ball screws incorporate dual collections of balls and are pressed over one another hence creating radial clearances close of 0 .

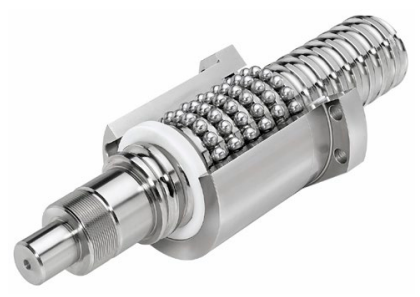

Fig 8: Circulating ball screw

The scheming of the length of screw is determined below. The travelling of xy table has been determined to be $400 \mathrm{~mm}$ by $350 \mathrm{~mm}$. In that case, the ball screw axis is $100 \mathrm{~mm}$ (allowances for bellow cover) $+200 \mathrm{~mm}$ (top board breadth) +400 $\mathrm{mm}$ (travel breadth) $=700 \mathrm{~mm}$. The screw length $=100 \mathrm{~mm}+200 \mathrm{~mm}+350 \mathrm{~mm}$ (allowances for bellow cover) (top arrangement) (travel) $=650 \mathrm{~mm}$. The commercialized ball screw is given from firms such as ABBA, Precision Bearing House, Grampus Impex Ltd and THK. The firms' specifications, which satisfy our necessities include BNF 200505 RRG2 850L 700-C7 from the THK firm.

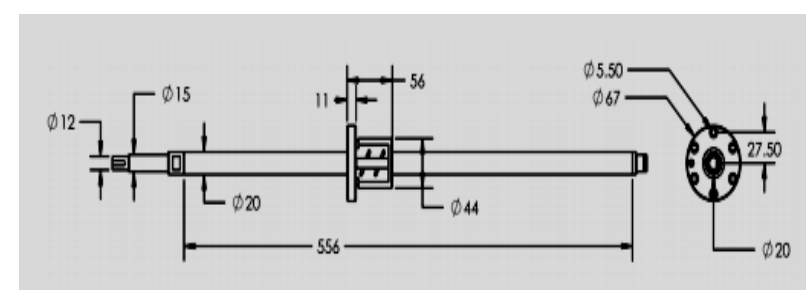

Fig 9: Machined ends and dimensions of the ball screw assembly

\section{B. Linear Motion Guidepath or LMGuidepath}

LMGuide rail (Fig 10) is applied for considerably precise Linear Motion (LM) [14]. It is capable to sustain higher loads in different paths and therefore can be mounted in different paths. The assembly incorporates the rails that guide the blocks in them. In these blocks, the roller and ball are available to significantly minimize the friction loss. In that case, LMGuidepath is considered in both robotics and firms to attain particular functions. 


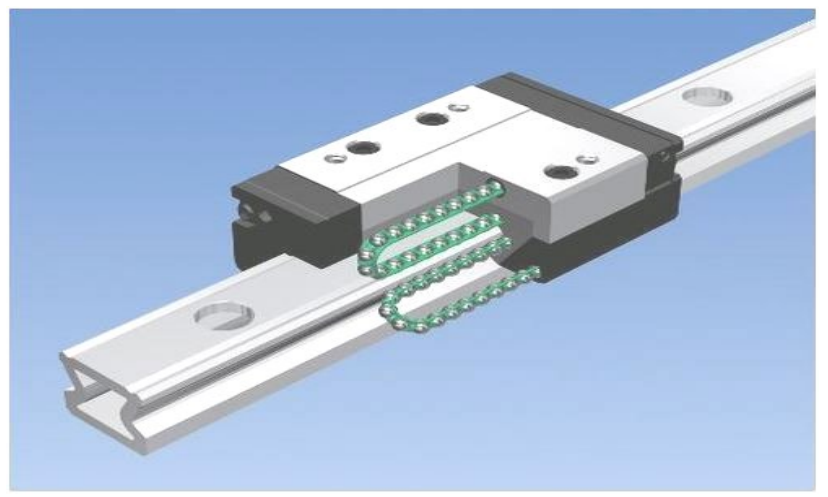

Fig 10: LMGuide rail assembly

The ball roll in four different rows of the race-path on LM rails and the blocks, including the end plates included in the LM blocks to allow for the circulation of balls. Because the retainer plate holds the ball, they do not fall over in case LM rail is pulled over. Every ball row is incorporated at the $45^{\circ}$ to allow the application of loads at the LM block. The loads are similar in four different paths (lateral, reverse, radial and reverse-radial paths). This enables LM guide to be utilized in the orientations. Moreover, LM block can accept the well-balanced preloads hence enhancing the strength in all the four paths whereas maintaining low and constant coefficients.

With the minimal sectional heights and the high rigidity designs of the blocks, this proposed model attains stable and accurate linear motion. The four-way load has each ball row placed in contact with the $45^{\circ}$ angle. Resultantly, this allows the rated load to be applied to LM blocks in all the four paths. In addition, it allows the LMGuide dimensions (Fig 11) to be utilized in the orientations and in wide-range; application is based on factors such as:

1) High Rigidity

Since the ball is arranged in four different rows in balanced manner, larger preloads are applied and rigidity in the various paths can be enhanced.

\section{2) High Durability}

Even based on biased load or preload, differential ball slip does not happen. Resultantly, long-lasting accuracy maintenance, high-wear resistance and smooth motion are attained.

\section{3) Multi-directional Rated Load}

The model can receive loads from all the paths. The basic load rate is the same in all the four paths and their actual values are provided in dimensional board in the model.

\section{4) Equivalent Loads}

Whenever LM blocks of the model accept the loads in lateral and radial-reverse paths concurrently, the corresponding loads are obtained from the calculation below:

$$
\mathrm{EL}=\mathrm{RD}(\mathrm{RL})+\mathrm{LD}
$$

Whereby EL represents the equivalent load $(\mathrm{N}) *$ Reverse-radial direction $(\mathrm{RD}) *$ radial direction $*$ Radial load $(\mathrm{RL})+$ Lateral Direction (LD)

a) LMGuide Advantages

- Higher speed operation

- High long-term precision

- Higher permissible load ratings

- High rigidity in various paths

- High precision with ease

- Smooth movements with minimal clearance

The combinations of these advantages produce minimal overall costs, higher precision in machines based on LMGuide, higher productivity, substantial power saving, high efficacy in the design of machines and simple maintenance.

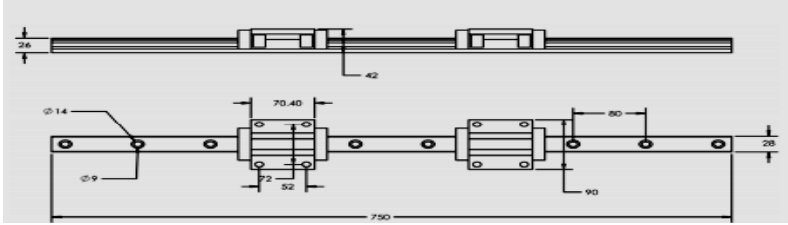

Fig 11: LMGuide dimensions 


\section{Jets}

The abrasive resources are based on the work surfaces at high speed via the jets [15]. In that case, the jets resources are subjected to extreme abrasive wear and therefore these have been structured using rigid resources such as synthetic sapphire and tungsten carbide. Tungsten carbide jets are utilized for circular cross-sections with a diameter ranging from $0.12 \mathrm{~mm}$ to $0.8 \mathrm{~mm}$, rectangular part of sizes $3.8 \mathrm{~mm}$ by $0.18 \mathrm{~mm}$ by $0.05 \mathrm{~mm}$ by $0.1 \mathrm{~mm}$ and the square segments of up to $0.75 \mathrm{~mm}$.

The sapphire jet is formed normally in parts. The part sizes range from $0.25 \mathrm{~mm}$ to $0.75 \mathrm{~mm}$ in diameter. The jet is structured with the outer taper structured to limit the subordinate effect because of abrasive ricocheting materials. The jet from tungsten carbide is assigned a mean life duration of about 29 hours and the sapphire jet is about 298 hours following the operations when used with $26 \mu \mathrm{m}$ AJM energy. MRR and the sizes of machined part are influenced by the distance of jets from the workpieces.

The abrasive resources from the jets pursue the parallel path for shorter distances before the jet flares hence amounting to oversized holes. It is witnessed that the jet streams is normally cylindrical and at about $1.6 \mathrm{~mm}$ and therefore flares into cone of $7^{\circ}$ angle. MRR initially develops with the advancement in the jets distance from the workpiece since accelerating particles leave the jets as shown in Fig 12. This increment develops to a distance of about $8 \mathrm{~mm}$ before it drops due to an enhanced machines region for the similar amount of abrasive and diminishes in speed of abrasive streams because of drags.

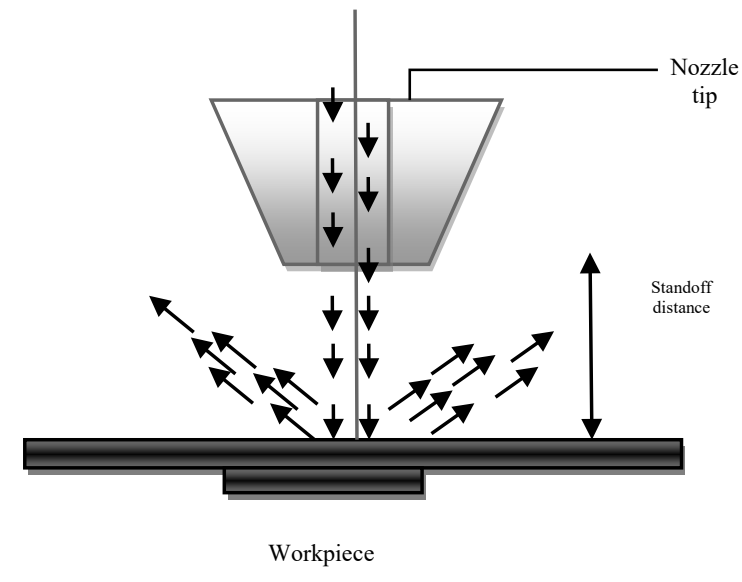

Fig 12: Abrasive behaviors of particles

\section{FINAL DESIGN PRESENTATION}

This section defines the final assembly drawings of AJM. The subsections reflect on the design limitations and the final presentation of the design.

\section{A. Design Limitations}

Irrespective of the simplified design, AJM can be challenging sometimes. There are various designs of AJM, but all these designs share the same issues. These issues incorporate: wearable misalignment and damaging of jewels; occasional mixing plugging tube: typically influenced by large and dirt abrasive particles; and shorter life of costly wear segments.

\section{B. Design Presentation}

It should be noted that the machines components such as piping, bellows cover, dehumidifier, vibrator and air compressor.

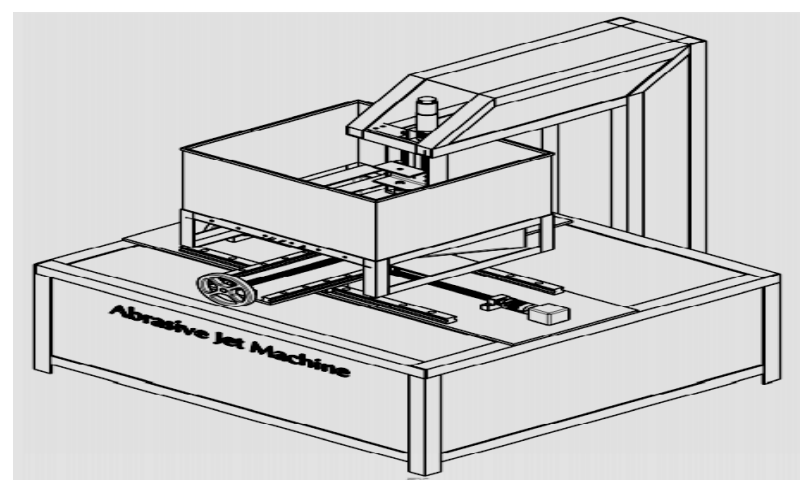

Fig 13: Final AJM design assembly (isometric view) 


\section{CONCLUSION AND FUTURE DIRECTIONS}

This paper recommends a design for AJM. The motion of xyz modules has been proposed considering the present machines components in the mechanical engineering industry. The assembling and designing of the model based on the wide-range components was a tiresome task; however, it was done in time. Nonetheless, since some parts could not be incorporated due to their costs, the final design assembly was typically limited to drilling processes. This machines proposal surpasses its present capability and position by incorporating automations to it. This has been achieved using DC servomotors and stepper motors with standardized standalone controllers, including 2D profiles changed to standardize Gcodes. However, future AJM designs should be develop to incorporate 3D profiles, which are changed to standard M-codes that are communicated to the AJM to perform the essential automations to enhance processes in engineering firms.

\section{References}

[1]. R. Tyagi, "A review of few unconventional machining processes based on the concept of velocity shear instability in plasma", Production \& Manufacturing Research, vol. 2, no. 1, pp. 216-227, 2014. Available: 10.1080/21693277.2014.899934.

[2]. M. Sugimoto, T. Shakouchi, K. Hayakawa, M. Okazaki and M. Izawa, "Particle Laden Impinging Jet Flow from Rectangular Jets and Abrasive Jet Machines(Multiphase Flow 2)", The Proceedings of the International Conference on Jets, Wakes and Separated Flows (ICJWSF), vol. 2005, no. 0, pp. 325-330, 2005. Available: 10.1299/jsmeicjwsf.2005.325.

[3]. Y. OKITA and K. KASHIWAGI, "Abrasive Suspension Jet Machines with Stabilized Abrasive Concentration", The Proceedings of Conference of Chugoku-Shikoku Branch, vol. 202058, no. 0, pp. 07a4, 2020. Available: 10.1299/jsmecs.2020.58.07a4.

[4]. "Calculations of material removal, removal rate, and Preston coefficient in continuous lapping / polishing machines", Precision Engineering, vol. 16, no. 3, p. 230, 1994. Available: 10.1016/0141-6359(94)90135-x.

[5]. R. Dhawan, N. Kawade and B. Dikshit, "Design and Performance of a Laser-Based Compact Position Sensor for Long Standoff Distance", IEEE Sensors Journal, vol. 18, no. 16, pp. 6557-6562, 2018. Available: 10.1109/jsen.2018.2849716.

[6]. Y. Ali, P. Mathew and J. Wang, "Progress in the Modeling of Abrasive Jet Machines", Advanced Materials Research, vol. 126-128, pp. 3-8, 2010. Available: 10.4028/www.scientific.net/amr.126-128.3.

[7]. M. Kaladhar, "Evaluation of hard coating materials performance on machinability issues and material removal rate during turning operations", Measurement, vol. 135, pp. 493-502, 2019. Available: 10.1016/j.measurement.2018.11.066.

[8]. J. Delgadillo and R. Rajamani, "Computational fluid dynamics prediction of the air-core in hydrocyclones", International Journal of Computational Fluid Dynamics, vol. 23, no. 2, pp. 189-197, 2009. Available: 10.1080/10618560902724893.

[9]. R. Li et al., "Pressure, Feed Rate, and Abrasive Mass Flow Rate Influence on Surface Roughness for Recombinant Bamboo Abrasive Water Jet Cutting", BioResources, vol. 10, no. 2, 2015. Available: 10.15376/biores.10.2.1998-2008.

[10]. J. Fair, "Development of High-Pressure Abrasive-Jet Drilling", Journal of Petroleum Technology, vol. 33, no. 08, pp. 1379-1388, 1981. Available: 10.2118/8442-pa.

[11]. P. Jeyapandiarajan and X. Anthony, "Evaluating the Machinability of Inconel 718 under Different Machines Conditions", Procedia Manufacturing, vol. 30, pp. 253-260, 2019. Available: 10.1016/j.promfg.2019.02.037.

[12]. H. Nouraei, A. Wodoslawsky, M. Papini and J. Spelt, "Characteristics of abrasive slurry jet micro-machines: A comparison with abrasive air jet micro-machines", Journal of Materials Processing Technology, vol. 213, no. 10, pp. 1711-1724, 2013. Available: 10.1016/j.jmatprotec.2013.03.024.

[13]. N. Yuvaraj and M. Pradeep Kumar, "Study and evaluation of abrasive water jet cutting performance on AA5083-H32 aluminum alloy by varying the jet impingement angles with different abrasive mesh sizes", Machines Science and Technology, vol. 21, no. 3, pp. 385-415, 2017. Available: $10.1080 / 10910344.2017 .1283958$.

[14]. S. Ramzan, "Crafting Linear Motion Problems for Problem- Based Learning Physics Classes", International Journal of Psychosocial Rehabilitation, vol. 24, no. 5, pp. 5426-5437, 2020. Available: 10.37200/ijpr/v24i5/pr2020249.

[15]. О. Вишневський, "Experimental models of monitoring abrasive wear surfaces of materials", Proceedings of National Aviation University, vol. 28, no. 2, 2006. Available: 10.18372/2306-1472.28.1342. 